\title{
Article \\ Effect of Initial Stress on an SH Wave in a Monoclinic Layer over a Heterogeneous Monoclinic Half-Space
}

\author{
Ambreen Afsar Khan ${ }^{1}$, Anum Dilshad ${ }^{1}$, Mohammad Rahimi-Gorji ${ }^{2, *}$ and Mohammad Mahtab Alam ${ }^{3}$ (D) \\ 1 Department of Mathematics and Statistics, International Islamic University, Islamabad 44000, Pakistan; \\ ambreen.afsar@iiu.edu.pk (A.A.K.); anumdilshad3@gmail.com (A.D.) \\ 2 Faculty of Medicine and Health Sciences, Ghent University, 9000 Ghent, Belgium \\ 3 Department of Basic Medical Sciences, College of Applied Medical Science, King Khalid University, \\ Abha 62529, Saudi Arabia; mmalam@kku.edu.sa \\ * Correspondence: mohammad.rahimigorji@ugent.be
}

Citation: Khan, A.A.; Dilshad, A.; Rahimi-Gorji, M.; Alam, M.M. Effect of Initial Stress on an SH Wave in a Monoclinic Layer over a Heterogeneous Monoclinic Half-Space. Mathematics 2021, 9, 3243. https://doi.org/10.3390/ math9243243

Academic Editor: Xiangmin Jiao

Received: 15 September 2021 Accepted: 11 December 2021 Published: 14 December 2021

Publisher's Note: MDPI stays neutral with regard to jurisdictional claims in published maps and institutional affiliations.

Copyright: (c) 2021 by the authors. Licensee MDPI, Basel, Switzerland. This article is an open access article distributed under the terms and conditions of the Creative Commons Attribution (CC BY) license (https:/ / creativecommons.org/licenses/by/ $4.0 /)$.

\begin{abstract}
Considering the propagation of an $\mathrm{SH}$ wave at a corrugated interface between a monoclinic layer and heterogeneous half-space in the presence of initial stress. The inhomogeneity in the halfspace is the causation of an exponential function of depth. Whittaker's function is employed to find the half-space solution. The dispersion relation has been established in closed form. The special cases are discussed, and the classical Love wave equation is one of the special cases. The influence of nonhomogeneity parameter, coupling parameter, and depth of irregularity on the phase velocity was studied.
\end{abstract}

Keywords: SH-wave; monoclinic; heterogeneous; undulatory; initial stress

\section{Introduction}

Many experimental and theoretical studies predicted that the Earth is a convoluted model in nature. Therefore, a more natural presentation of the medium through which seismic waves propagate is required. The propagation of a wave in an inhomogeneous medium is of keen interest due to the continuous variation in the elastic properties of the material. The heterogeneity in the material is produced by a change in rigidity and density. Various authors have taken various forms of the variation, like linear exponential, quadratic, etc., for simulating the variation in different geological parameters inside the Earth. Jeffrey [1] deliberated the impact of heterogeneity on the Love wave. Bullen [2] mentioned that density varies inside the Earth at different rates. Wilson [3] examined the propagation of the Rayleigh wave in a heterogeneous medium. Dhua and Chattopadhyay [4] discussed wave propagation in heterogeneous layers of the Earth. Alam et al. [5] examined the propagation of an $\mathrm{SH}-$ wave in an anisotropic crustal layer over a heterogeneous half-space. Taking into account the structure and characteristics of the Earth, a variety of crustal forms are possible. Crystals are solid in nature and bounded by faces or plane surfaces. The monoclinic form is one of them. It has three unequal axes, two intersecting at an oblique angle, and the third is transverse to them. Several authors worked in this direction [6-12].

Initial stress develops in the body due to many physical causes. The initial stress has a great impact on the rigidity of the elastic structure and produces a mechanical fault called buckling. Biot [13] firstly examined the propagation of light under initial stress. A detailed study on the propagation of Love, Rayleigh, and $\mathrm{SH}-$ waves in a pre-stress heterogeneous half-space was made by Chaterjee et al. [14]. Singh et al. [15] analyzed the impact of stress and irregularity on the Love wave in a heterogeneous medium. They have shown that horizontal stress has a favorable impact on the phase velocity. Abd-Alla et al. [16] discussed the impact of 
stress on an SV wave at the solid-liquid interface. Verma et al. [17] examined the influence of initial stress on the propagation of the Rayleigh wave in a heterogeneous medium. Khan and Afzal [18] examined the impact of initial stress on the viscoelastic liquid. They found that the reflected and refracted $\mathrm{P}$ wave has a maximum amplitude ratio.

Motivated in this paper by the above studies, we have considered the propagation of an $\mathrm{SH}$-wave in a homogeneous monoclinic layer lying over a heterogeneous half-space. Initial stress, rigidity, and density are assumed to vary with depth. The dispersion curves are predicted by graphs for various values of non-homogeneity parameters and different sizes of irregularity.

\section{Problem Formulation}

Let us assume the Cartesian coordinate system; hence a wave propagating along $x$-axis and $z$-axis is considered vertically downward. A nonhomogeneous monoclinic layer $N_{1}$ : $\left[r_{1}(x)-h \leq z \leq r_{2}(x)\right]$ over a heterogeneous monoclinic half-space $N_{2}:\left[r_{2}(x) \leq z \leq \infty\right]$, where $r_{1}(x)$ and $r_{2}(x)$ is periodic, and continuous functions of $x$ and $h$ is the thickness of the layer. The Fourier series of these functions are written as

$$
r_{j}(x)=\sum_{n=1}^{\infty}\left[r_{n}^{j} e^{i n \alpha x}+r_{-n}^{j} e^{-i n \alpha x}\right],
$$

where $r_{n}{ }^{j}$ and $r_{-n}{ }^{j}$ are $n$th order coefficient of Fourier series expansion

$$
r_{\mp n}^{j}=\left\{\begin{array}{c}
\frac{a_{j}}{2} \text { for } n=1 j=1,2, \\
\frac{S_{n}^{j} \mp Q_{n}^{j}}{2}, \text { for } n=2,3,4, \ldots
\end{array}\right.
$$

where $S_{n}^{j}$ and $Q_{n}^{j}$ are coefficient of Fourier series expansion.

Equation (1) can be explained as follow (Ref. [19])

$$
r_{1}=a_{1} \cos \alpha x, r_{2}=a_{2} \cos \alpha x,
$$

where $\alpha$ the wave is number, $a_{1}$ and $a_{2}$ are the amplitudes of the upper and wavy surface.

\section{SH-Wave in Homogeneous Monoclinic Layer}

We consider the equation of motion in the presence of initial stress $P$ and magnetic field (Ref. [6]).

$$
\tau_{i j, j}-\frac{P}{2} \frac{\partial^{2} v_{1}}{\partial x^{2}}+(\vec{J} \times \vec{B})_{i}=\rho_{1} \frac{\partial^{2} U_{i}}{\partial t^{2}}, \quad i, j=1,2,3
$$

where $U_{i}=\left[u_{1}, v_{1}, w_{1}\right]$ is the displacement vector, $\rho_{1}$ the mass density, $\vec{B}$ the magnetic induction vector, and $\vec{J}$ the electric current density.

The stress-strain relation is defined as

$$
\tau_{i j}=C_{i j k l} e_{k l}
$$

We consider the homogeneity of the monoclinic layer as,

$$
\begin{gathered}
C_{12}=C_{12}, C_{11}=C_{11}, C_{13}=C_{13}, C_{25}=C_{25}, \\
C_{15}=C_{15}, C_{23}=C_{23}, C_{46}=C_{46}, C_{33}=C_{33}, \\
C_{44}=C_{44}, C_{35}=C_{35}, C_{66}=C_{66}, \\
C_{55}=C_{55}, C_{22}=C_{22} .
\end{gathered}
$$


The stress-strain relations for are given by:

$$
\begin{gathered}
\tau_{11}=C_{11} e_{11}+C_{21} e_{22}+C_{13} e_{33}+C_{15} e_{13}, \\
\tau_{22}=C_{21} e_{11}+C_{22} e_{22}+C_{23} e_{33}+C_{25} e_{13}, \\
\tau_{33}=C_{13} e_{11}+C_{23} e_{22}+C_{33} e_{33}+C_{35} e_{13}, \\
\tau_{23}=C_{44} e_{23}+C_{46} e_{12}, \\
\tau_{13}=C_{15} e_{11}+C_{25} e_{22}+C_{35} e_{33}+C_{55} e_{13}, \\
\tau_{12}=C_{44} e_{23}+C_{66} e_{12},
\end{gathered}
$$

where $C_{i j}=C_{j i}(i, j=1,2, \ldots \ldots, 6)$ are elastic constants and

$$
e_{i j}=\frac{1}{2}\left(\frac{\partial u_{i}}{\partial x_{j}}+\frac{\partial u_{j}}{\partial x_{i}}\right)
$$

When the SH-wave is propagating in $x z$ plane, we have the following components of displacement

$$
v_{1}=v_{1}(x, z, t), \quad u_{1}=0=w_{1} .
$$

Using Equation (7) into Equation (3), we have

$$
\frac{\partial \tau_{12}}{\partial x}+\frac{\partial \tau_{23}}{\partial z}-\frac{P}{2} \frac{\partial^{2} v_{1}}{\partial x^{2}}+(\vec{J} \times \vec{B})_{2}=\rho_{1} \frac{\partial^{2} v_{1}}{\partial t^{2}}
$$

Maxwell's equations are

$$
\vec{\nabla} \cdot \vec{B}=0, \vec{\nabla} \times \vec{E}=-\frac{\partial \vec{B}}{\partial t}, \quad \vec{\nabla} \cdot \vec{E}=\frac{\rho_{e}}{\epsilon}, \quad \vec{B}=\mu_{e} \vec{H}, \quad \vec{\nabla} \times \vec{H}=\vec{J}
$$

where $\vec{E}$ is the electric field, $\vec{H}$ includes both the induced and primary magnetic fields, $\rho_{e}$ is charge density, $\mu_{e}$ is permeability, $\epsilon$ is the permittivity of free space, and $\sigma$ is the conduction coefficient. Then Maxwell's stress tensor $\left(\tau_{\mathrm{ij}}\right)^{M}$ is given by Ref. [18]

$$
\left(\tau_{\mathrm{ij}}\right)^{M}=\mu_{e}\left[H_{i} h_{j}+H_{j} h_{i}-H_{k} h_{k} \delta_{\mathrm{ij}}\right],
$$

where $h_{i}=\left[h_{1}, h_{2}, h_{3}\right]$ is the change in the magnetic field.

In the absence of displacement current Equation (9) becomes

$$
\frac{\nabla^{2} \vec{H}}{\sigma \mu_{e}}=\left[\frac{\partial \vec{H}}{\partial t}-\vec{\nabla} \times\left(\frac{\partial \vec{U}}{\partial t} \times \vec{H}\right)\right]
$$

Components form of Equation (11) is given below

$$
\frac{\partial H_{x}}{\partial t}=\frac{1}{\sigma \mu_{e}} \nabla^{2} H_{x}, \frac{\partial H_{y}}{\partial t}=\frac{1}{\sigma \mu_{e}} \nabla^{2} H_{y}+\left[\frac{\partial}{\partial x}\left(H_{x} \frac{\partial v_{1}}{\partial \mathrm{t}}\right)+\frac{\partial}{\partial z}\left(H_{z} \frac{\partial v_{1}}{\partial \mathrm{t}}\right)\right], \frac{\partial H_{z}}{\partial t}=\frac{1}{\sigma \mu_{e}} \nabla^{2} H_{y} .
$$

When $\sigma \rightarrow \infty$ Equation (12) becomes

$$
\frac{\partial H_{x}}{\partial t}=0=\frac{\partial H_{z}}{\partial t}
$$

and

$$
\frac{\partial H_{y}}{\partial t}=\left[\frac{\partial}{\partial x}\left(H_{x} \frac{\partial v_{1}}{\partial \mathrm{t}}\right)+\frac{\partial}{\partial z}\left(H_{z} \frac{\partial v_{1}}{\partial \mathrm{t}}\right)\right]
$$


Equation (13) shows that $H_{x}$ and $H_{z}$ have no perturbation, but Equation (14) indicates there may be some perturbation in $H_{y}$. Hence there is some perturbation $h_{2}$ in $H_{y}$, the only component of an induced magnetic field. The components of the magnetic field can be written as

$$
H_{x}=H_{01}, \quad H_{y}=H_{02}+h_{2}, \quad H_{z}=H_{03}
$$

Initially the $h_{2}$ is zero. The magnetic field is normal to the polarization of the wave, i.e., $H_{02}=0$. The wave crosses the primary magnetic field at an angle $\Phi$.

$$
\begin{aligned}
& \vec{H}_{0}=\left(H_{0} \cos \Phi, 0, H_{0} \sin \Phi\right), \\
& \vec{H}=\left(H_{0} \cos \Phi, h_{2}, H_{0} \sin \Phi\right) .
\end{aligned}
$$

Using Equation (16) into Equation (14), one obtains

$$
\frac{\partial h_{2}}{\partial \mathrm{t}}=\frac{\partial}{\partial \mathrm{t}}\left[H_{0} \cos \Phi \frac{\partial v_{1}}{\partial \mathrm{x}}+H_{0} \sin \Phi \frac{\partial v_{1}}{\partial \mathrm{z}}\right] .
$$

Integrating on both sides, we get

$$
h_{2}=H_{0} \cos \Phi \frac{\partial v_{1}}{\partial \mathrm{x}}+H_{0} \sin \Phi \frac{\partial v_{1}}{\partial \mathrm{z}} .
$$

Now $\vec{J} \times \vec{B}$ becomes

$$
\vec{J} \times \vec{B}=\mu_{e}\left[(\vec{H} \cdot \vec{\nabla}) \vec{H}-\frac{1}{2} \vec{\nabla} \vec{H}^{2}\right] .
$$

Putting the values of $\vec{J} \times \vec{B}, \tau_{12}$ and $\tau_{23}$ in Equation (8), we have

$$
L \frac{\partial^{2} v_{1}}{\partial x^{2}}+2 M \frac{\partial^{2} v_{1}}{\partial x \partial z}+N \frac{\partial^{2} v_{1}}{\partial z^{2}}=\rho_{1} \frac{\partial^{2} v_{1}}{\partial t^{2}}
$$

where $m=\frac{\mu_{e} H_{0}{ }^{2}}{C_{44}}$, the coupling parameter of magnetoelastic medium,

$$
\begin{gathered}
L=C_{44}\left(O-\frac{p}{2 C_{44}}\right), O=C_{44}\left(\frac{C_{66}}{C_{44}}+m \cos ^{2} \Phi\right), \\
M=C_{44}\left(\frac{C_{46}}{C_{44}}+m \cos \Phi \sin \Phi\right), N=C_{44}\left(1+m \sin ^{2} \Phi\right) .
\end{gathered}
$$

A harmonic wave traveling in the positive $x$ direction.

$$
v_{1}=V_{1}(z) e^{i k(x-c t)},
$$

Substituting Equation (21) into Equation (20), we have

$$
\frac{d^{2} V_{1}(z)}{d z^{2}}+\eta_{1} \frac{d V_{1}}{d z}+\eta_{2} V_{1}(z)=0
$$

where,

$$
\eta_{1}=2 i k \frac{M}{N}, \eta_{2}=k^{2}\left(\frac{c^{2}}{c^{2}{ }_{1}}-\frac{L}{N}\right), q=\frac{\sqrt{\eta_{1}^{2}-4 \eta_{2}}}{2}, c_{1}=\sqrt{\frac{N}{\rho}} .
$$

The solution of Equation (23) is given below

$$
v_{1}(z)=e^{-\frac{\eta_{1}}{2} z}(A \cos q z+B \sin q z) e^{i k(x-c t)} .
$$




\section{SH-Wave in Heterogeneous Monoclinic Half Space}

Consider heterogeneous monoclinic half-space as

$$
\begin{gathered}
C_{21}(z)=C_{21} e^{v z}, C_{11}(z)=C_{11} e^{v z}, C_{13}(z)=C_{13} e^{v z}, C_{25}(z)=C_{25} e^{v z}, \\
C_{15(z)=} C_{15} e^{v z}, C_{23}(z)=C_{23} e^{v z}, C_{46}(z)=C_{46} e^{v z}, C_{33}(z)=C_{33} e^{v z}, \\
C_{44}(z)=C_{44} e^{v z}, C_{35}(z)=C_{35} e^{v z}, C_{66}(z)=C_{66} e^{v z}, \\
C_{55}(z)=C_{55} e^{v z}, C_{22}(z)=C_{22} e^{v z},
\end{gathered}
$$

where $v$ is the real heterogeneous constants.

The stress-strain relations for heterogeneous medium is given as

$$
\begin{gathered}
\tau_{11}=C_{11} e_{11} e^{v z}+C_{21} e_{22} e^{v z}+C_{13} e_{33} e^{v z}+C_{15} e_{13} e^{v z}, \\
\tau_{22}=C_{21} e_{11} e^{v z}+C_{22} e_{22} e^{v z}+C_{23} e_{33} e^{v z}+C_{25} e_{13} e^{v z}{ }^{v z}, \\
\tau_{33}=C_{13} e_{11} e^{v z}+C_{23} e_{22} e^{v z}+C_{33} e_{33} e^{v z}+C_{35} e_{13} e^{v z}, \\
\tau_{23}=C_{44} e_{23} e^{v z}+C_{46} e_{12} e^{v z}, \\
\tau_{13}=C_{15} e_{11} e^{v z}+C_{25} e_{22} e^{v z}+C_{35} e_{33} e^{v z}+C_{55} e_{13} e^{v z}, \\
\tau_{12}=C_{44} e_{23} e^{v z}+C_{66} e_{12} e^{v z},
\end{gathered}
$$

where $C_{i j}=C_{j i}(i, j=1,2, \ldots \ldots, 6)$ are elastic constants and

$$
e_{i j}=\frac{1}{2}\left(\frac{\partial u_{i}}{\partial x_{j}}+\frac{\partial u_{j}}{\partial x_{i}}\right)
$$

Now, we consider the equation of motion in the presence of initial stress $P$ and in the $x$ direction and in the absence of a magnetic field is given as

$$
\begin{gathered}
\tau_{i j, j}-\frac{P}{2} \frac{\partial^{2} v_{2}}{\partial x^{2}}=\rho \frac{\partial^{2} v_{2}}{\partial t^{2}}, \quad i, j=1,2,3, \\
v_{2}=v_{2}(x, z, t), \quad u_{2}=0=w_{2}, \frac{\partial}{\partial y}=0
\end{gathered}
$$

For heterogeneous medium initial stress, $P$ and density $\rho$ are explained as Ref. [5]

$$
\rho=\rho^{\prime} e^{\alpha z}, P=p^{\prime} e^{\beta z},
$$

where $\alpha$ and $\beta$ are real heterogeneous constants. Using Equations (26), (28), and (29) into Equation (27), it takes the following form

$\frac{\partial}{\partial x}\left[\left(C_{46} \frac{\partial v_{2}}{\partial z}\right)+\left(C_{66}-\frac{p^{\prime}}{2}\right) \frac{\partial v_{2}}{\partial x}\right]+\frac{\partial}{\partial z}\left[C_{46} \frac{\partial v_{2}}{\partial x}+C_{44} \frac{\partial v_{2}}{\partial z}+v\left(C_{46} \frac{\partial v_{2}}{\partial x}+C_{44} \frac{\partial v_{2}}{\partial z}\right)\right]=\rho^{\prime} \frac{e^{\beta z}}{e^{\alpha z}} \frac{\partial^{2} v_{2}}{\partial t^{2}}$.

Let us assume a harmonic wave traveling in the positive $x$ direction.

$$
v_{2}=V_{2}(z) e^{i k(x-c t)} \text {. }
$$

Using Equation (31) into Equation (30), we get

$$
\frac{d^{2} V_{2}(z)}{d z^{2}}+\zeta_{1} \frac{d V_{2}}{d z}-k^{2}\left(\zeta_{2}-\frac{c^{2}}{c^{2}} \frac{e^{\beta z}}{e^{\alpha z}}\right) V_{2}(z)=0
$$

where $\zeta_{1}=\frac{2 i k C_{46}+v C_{44}}{C_{44}}, \zeta_{2}=\frac{v i C_{46}}{k C_{44}}+C_{66}-\frac{p^{\prime}}{2}, c_{0}=\sqrt{\frac{C_{44}}{\rho^{\prime}}}$.

Using $V_{2}(z)=\psi(z) e^{\frac{-\alpha z}{2}}$ into Equation (32), we get simplified form as below

$$
\frac{d^{2} \psi(z)}{d z^{2}}+\left[\frac{k^{2} c^{2}(1+\beta z)}{c^{2}{ }_{0}(1+\alpha z)}-k^{2}\left(\zeta_{2}+\frac{\alpha \zeta_{1}}{k^{2}}+\frac{\alpha^{2}}{4 k^{2}}\right) \psi(z)\right]=0 .
$$


The quantities are defined as below

$$
\begin{gathered}
\gamma=\sqrt{\zeta_{2}-\frac{\beta c^{2}}{\alpha c_{0}^{2}}+\frac{\alpha^{2}}{4 k^{2}}+\frac{\alpha \zeta_{1}}{k^{2}},} \\
z=\frac{\Omega}{2 k \gamma}-\frac{1}{\alpha}, \Omega=\frac{2 k \gamma}{\alpha}(1+\alpha z), \\
\frac{d^{2} \psi(\Omega)}{d \varphi^{2}}+\left(\frac{R}{\Omega}-\frac{1}{4}\right) \psi(\Omega)=0,
\end{gathered}
$$

where $R=\frac{k^{2} c^{2}(\alpha-\beta)}{2 \gamma c_{0}^{2} \alpha^{2}}$

Equation (36) is the Whittaker equation [20]. The solution of Equation (36) is written as

$$
\psi(\Omega)=C W_{R, \frac{1}{2}}(\Omega)+D W_{-R, \frac{1}{2}}(\Omega),
$$

where $C$ and $\mathrm{D}$ are arbitrary constants, $W_{R, \frac{1}{2}}(\Omega)$ and $W_{-R, \frac{1}{2}}(\Omega)$ are Whittaker's functions. $\lim _{z \rightarrow \infty} \psi(\Omega) \rightarrow 0$ and $\lim _{z \rightarrow \infty} V_{1}(z) \rightarrow 0$ Then the approximate required solution of inhomogeneous half-space is

$$
v_{2}=C W_{R, \frac{1}{2}}(\Omega) e^{-\frac{\alpha z}{2}} e^{i k(x-c t)} .
$$

Consider the first two terms of Whittaker's function $W_{R, \frac{1}{2}}(\Omega)$, then the Equation (38)

$$
v_{2}=C \Omega e^{-\frac{\Omega}{2}}\left[1+\frac{(1-R)}{2} \Omega\right] e^{-\frac{\alpha z}{2}} e^{i k(x-c t)} .
$$

\section{Boundary Conditions}

We consider the following boundary conditions.

Stresses at the interference is

$$
\left[\tau_{23}+\left(\tau_{23}\right)^{M}\right]-r_{1}^{\prime}\left[\tau_{12}+\left(\tau_{12}\right)^{M}\right]=0 \text {, at } z=r_{1}(x)-h,
$$

After substituting the expression of $\sigma_{23},\left(\sigma_{23}\right)^{M}$ into the above expression, we get

$$
\left(N \frac{\partial v_{1}}{\partial z}+M \frac{\partial v_{1}}{\partial x}\right)-r_{1}{ }^{\prime}\left(M \frac{\partial v_{1}}{\partial x}+O \frac{\partial v_{1}}{\partial z}\right)=0
$$

Stresses are continuous at the common corrugated interference.

$$
\begin{gathered}
{\left[\tau_{23}+\left(\tau_{23}\right)^{M}\right]-r_{2}{ }^{\prime}\left[\tau_{12}+\left(\tau_{12}\right)^{M}\right]=\tau_{23}-r_{2}{ }^{\prime} \tau_{12} \text { at } \mathrm{z}=r_{2}(x)} \\
\left.M \frac{\partial v_{1}}{\partial z}-r_{2}^{\prime} O \frac{\partial v_{1}}{\partial x}=e^{v z}\left[\left(C_{44}-r_{2}{ }^{\prime} C_{46}\right) \frac{\partial v_{2}}{\partial z}\right)+\left(C_{46}-r_{2}{ }^{\prime} C_{66}\right) \frac{\partial v_{2}}{\partial x}\right]
\end{gathered}
$$

Displacements are continuous at the common corrugated interference.

$$
v_{1}=v_{2}, \text { at } \mathrm{z}=r_{2}(x) \text {. }
$$

\section{Dispersion Relation}

Using the solution in Equations (24) and (39) into the boundary conditions, we get the dispersion equation as 


$$
\begin{aligned}
& A\left[\left(i k g_{2}-g_{1} \frac{\eta_{1}}{2}\right) \cos q\left(r_{1}-h\right)-g_{1} q \sin q\left(r_{1}-h\right)\right]+B\left[\left(i k g_{2}-g_{1} \frac{\eta_{1}}{2}\right) \sin q\left(r_{1}-h\right)+g_{1} q \cos q\left(r_{1}-h\right)\right]=0 \\
& A\left[\left(i k s_{2}-s_{1} \frac{\eta_{1}}{2}\right) \cos q r_{2}-s_{1} q \sin q r_{2}\right]+B\left[\left(i k s_{2}-s_{1} \frac{\eta_{1}}{2}\right) \operatorname{sinq} r_{2}+s_{1} q \cos q r_{2}\right]+C X=0 \\
& \cos q r_{2}+B \operatorname{sinq} r_{2}+C S=0
\end{aligned}
$$

where

$$
\begin{gathered}
g_{1}=N-r_{1}{ }^{\prime} M, \quad s_{1}=N-r_{2}{ }^{\prime} M, \\
g_{2}=M-r_{1}{ }^{\prime} O, \quad s_{2}=M-r_{2}{ }^{\prime} O, \\
S=-\frac{2 k \gamma}{\alpha} e^{-\left[\frac{k \gamma}{\alpha}+\left(k \gamma+\frac{\alpha}{2}\right) r_{2}\right]}\left\{\left(1+\alpha r_{2}\right)+(1-R)\left(1+\alpha r_{2}\right)^{2} \frac{\gamma k}{\alpha}\right\} e^{-\frac{\eta_{1}}{2} r_{2},}
\end{gathered}
$$$$
\begin{aligned}
X= & -\frac{2 k \gamma}{\alpha}\left\{1+(1-R)\left(1+\alpha r_{2}\right) \frac{\gamma k}{\alpha}\right\} e^{-\left[\frac{k \gamma}{\alpha}+\left(k \gamma+\frac{\alpha}{2}\right) r_{2}\right]} e^{\left(\frac{\eta_{1}}{2}+\mathrm{v}+\alpha\right) r_{2}}\left\{( 1 + \alpha r _ { 2 } ) \left[\left(C_{44}-\right.\right.\right. \\
& \left.\left.\left.r_{2}^{\prime} C_{46}\right)\left[\left(k \gamma+\frac{\alpha}{2}\right)-\frac{\alpha}{\left(1+\alpha \lambda_{2}\right)}-\frac{(1-R) \gamma k}{1+(1-R)\left(1+\alpha r_{2}\right) \frac{\gamma k}{\alpha}}\right]\right]+i k\left(C_{44}-r_{2} C_{46}\right)\right\},
\end{aligned}
$$

$$
T=\left[\left(C_{44}-r_{2}{ }^{\prime} C_{46}\right)\left[\left(k \gamma+\frac{\alpha}{2}\right)-\frac{\alpha}{\left(1+\alpha \lambda_{2}\right)}-\frac{(1-R) \gamma k}{1+(1-R)\left(1+\alpha r_{2}\right) \frac{\gamma k}{\alpha}}\right],\right.
$$

$$
\operatorname{Tank}\left(r_{2}-r_{1}+h\right) \sqrt{\frac{c^{2}}{c_{1}^{2}}-\frac{L}{N}+\left(\frac{M}{N}\right)^{2}}=\frac{s T \sqrt{\frac{c^{2}}{c_{1}{ }^{2}}-\frac{L}{N}+\left(\frac{M}{N}\right)^{2}}\left[r_{2}{ }^{2}-2 r_{1} r_{2} \frac{M}{N}-r_{1}{ }^{2}\left(\frac{c^{2}}{c_{1}{ }^{2}}-\frac{L}{N}\right)\right] e^{(\alpha+v) r_{2}}}{\left(s_{2} g_{2}-g_{1} s_{1} \frac{M}{N}-g_{2} s_{1} \frac{M}{N}-g_{1} s_{1}\left(\frac{c^{2}}{c_{1}{ }^{2}}-\frac{L}{N}\right)\right)^{2}+\left(T\left[g_{2}-g_{1} \frac{M}{N}\right] e^{(\alpha+v) r_{2}}\right)^{2}}
$$

Equation (43) is the dispersion relation for an $\mathrm{SH}$-wave in a monoclinic layer over a heterogeneous half-space.

\section{Particular Case}

\section{Case 1: Upper free surface is planar}

When upper free surface, i.e., $r_{1}=0$ and the lower surface is corrugated $r_{2}=a_{2} \cos (\alpha x)$, then the dispersion relation (43) can be reduced

$$
\operatorname{Tank}\left[h+a_{2} \cos \alpha x\right] \sqrt{\frac{c^{2}}{c_{1}^{2}}-\frac{L}{N}+\left(\frac{M}{N}\right)^{2}}=\frac{\mu_{1}\left[\gamma+\frac{\alpha}{2}-\frac{\alpha}{1+\alpha a_{2} \cos (\alpha x)}-\frac{(1-R) \gamma e^{(\alpha+v) a_{2} \cos (\alpha x)}}{\left.1+(1-R) 1+\alpha a_{2} \cos (\alpha x)\right) \frac{\gamma k}{a}}\right]}{v \sqrt{\frac{c^{2}}{c_{1}^{2}}-\frac{L}{N}+\left(\frac{M}{N}\right)^{2}}}
$$

where $v=\left[N+a a_{2} M \operatorname{Sin}(\alpha x)\right]$,

\section{Case 2: Lower common surface is planar}

When the lower common surface is planar, i.e., $r_{2}=0$ and the upper free surface is wavy $r 1=a_{1} \cos \alpha x$, then the dispersion relation (43) can be reduced into

$$
\begin{aligned}
& \operatorname{Tank}\left[h+a_{1} \cos \alpha x\right] \sqrt{\frac{c^{2}}{c_{1}{ }^{2}}-\frac{L}{N}+\left(\frac{M}{N}\right)^{2}}=\frac{N\left[\gamma+\frac{\alpha}{2}-\alpha+\frac{(1-R) \gamma}{1+(1-R) \frac{\gamma k}{a}}\right] \sqrt{\frac{c^{2}}{c_{1}{ }^{2}}-\frac{L}{N}+\left(\frac{M}{N}\right)^{2}}\left[\bar{g}_{2}{ }^{2}-2 \bar{g}_{1} \bar{g}_{2} \frac{L}{N}-\bar{g}_{1}{ }^{2}\left(\frac{c^{2}}{c_{1}{ }^{2}}-\frac{L}{N}\right)\right]}{\left(\bar{g}_{1} \frac{M^{2}}{N}+\bar{g}_{1} N\left(\frac{c^{2}}{c_{1}{ }^{2}}-\frac{L}{N}\right)\right)^{2}+\left(\mu_{1} \gamma+\frac{\alpha}{2}+\alpha-\frac{(1-R)}{1+(1-R) \frac{\gamma k}{a}}\left(\bar{g}_{2}-\bar{g}_{1} \frac{M}{N}\right)\right)^{2}}, \\
& \bar{g}_{1}=\left[N+a a_{1} M \sin (\alpha x)\right], \\
& \overline{g_{2}}=\left[M+a a_{1} O \sin (\alpha x)\right],
\end{aligned}
$$




\section{Case 3: Both Corrugations have Equal Amplitudes}

For equal amplitudes of both corrugations, i.e., $a_{1}=a_{2}=\mathrm{d} \Rightarrow r_{1}=r_{2}=d \cos \alpha x$, then the dispersion relation (43) can be changes into

$$
\begin{gathered}
\text { Tankh } \sqrt{\frac{c^{2}}{c_{1}{ }^{2}}-\frac{L}{N}+\left(\frac{M}{N}\right)^{2}}=\frac{g^{2} \mu_{1} \bar{T} \sqrt{\frac{c^{2}}{c_{1}{ }^{2}}-\frac{L}{N}+\left(\frac{M}{N}\right)^{2}}\left[s\left(\frac{s}{g}-2 \frac{M}{N}\right)-r\left(\frac{c^{2}}{c_{1}{ }^{2}}-\frac{L}{N}\right)\right] e^{(\alpha+v)[d \cos (\alpha x)]}}{\left[s_{2}{ }^{2}-2 g s \frac{M}{N}-r^{2}\left(\frac{c^{2}}{c_{1}{ }^{2}}-\frac{L}{N}\right)\right]^{2}+\left[\mu_{1} \bar{T}\left(s-r \frac{N}{0}\right) e^{(\alpha+v)[\operatorname{dcos}(\alpha x)]]^{2}}\right.}, \\
\bar{T}=\left(k \gamma+\frac{\alpha}{2}\right)-\frac{\alpha}{(1+d \cos (\alpha x))}-\frac{(1-R) \gamma k}{1+(1-R)\left(1+d \cos (\alpha x) \frac{\gamma k}{\alpha}\right.} .
\end{gathered}
$$

\section{Case 4: Isotropic Media with Planar Boundary Surface}

If the layer is isotropic and neglected the magnetic field, and half-space is uniform isotropic, i.e., $r_{1}=r_{2}=0, m=0, C_{44}=0, C_{46}=0, \alpha \rightarrow 0, \beta \rightarrow 0$, or $\mathrm{p} \rightarrow 0$, then Equation (43) can be reduced into the Love wave equation.

$$
\operatorname{Tan}\left[k h \sqrt{\left(\frac{c^{2}}{c_{1}^{2}}-1\right)}\right]=\frac{\sqrt{1-\frac{c^{2}}{c_{0}^{2}}}}{\sqrt{\frac{c^{2}}{c_{1}^{2}}-1}} .
$$

\section{Numerical Results}

For monoclinic layer the data is taken from Kumer et al. [9] as follows

$$
C_{44}=94 \mathrm{Gpa}, C_{46}=-11 \mathrm{Gpa}, C_{66}=93 \mathrm{Gpa} .
$$

For monoclinic half-space, we have used data given in Gubbin [21]

$$
\rho_{1}=7450 \frac{\mathrm{kg}}{\mathrm{m}^{3}}, \rho_{2}=3321 \frac{\mathrm{kg}}{\mathrm{m}^{3}}, \mu_{e}=71 \mathrm{Gpa}
$$

The dimensionless parameter called elastic coupling parameter $m=\frac{\mu_{e} H_{0}{ }^{2}}{C_{44}}$, heterogeneities parameters $(\alpha, \beta)$, position parameters, $(h)$ initial stress parameter $(p)$ has been numerically and graphically evaluated for their different values. Now we take an angle $\phi$ be fixed $10^{0}$ in all figures. The effect of coupling parameter $m$ can be seen in Figure 1 . We have noticed that the influence of the coupling parameter increases the phase velocity. Figures 2 and 3 show the effect of heterogeneous parameters $(\alpha$ and $\beta)$. In both figures, we perceived that the phase velocity of SH-wave declines as the value of $(\alpha$ and $\beta$ ) increases. More energy is released by the waves when the inhomogeneity of the medium is increased. That is why the phase velocity decreases. Figure 4 is a graphical representation of initial stress. We noticed that the phase velocity declines at initial stress. Figure 5 indicates the variation of position parameter $h$ on the phase velocity. The phase velocity decreases as the position parameter rises. Figures 6 and 7 show the impact of corrugation on the phase velocity. Figure 8 is related to case I, wherein $a_{1}=0$. These figures indicate that the phase velocity rises uniformly as $a_{1}$ rises. Figures 8 and 9 display the impact of common corrugation parameter $a_{2}$ on the phase velocity. The curve of Figure 9 is related to case II. These figures explain that the phase velocity decreases uniformly as the value of $a_{2}$ rises. All figures satisfied the fact that the phase velocity of $\mathrm{SH}$ waves declines with wave number. The results are obtained by using the basic condition of $\mathrm{SH}$ wave $c_{1}<c<c_{2}$. 


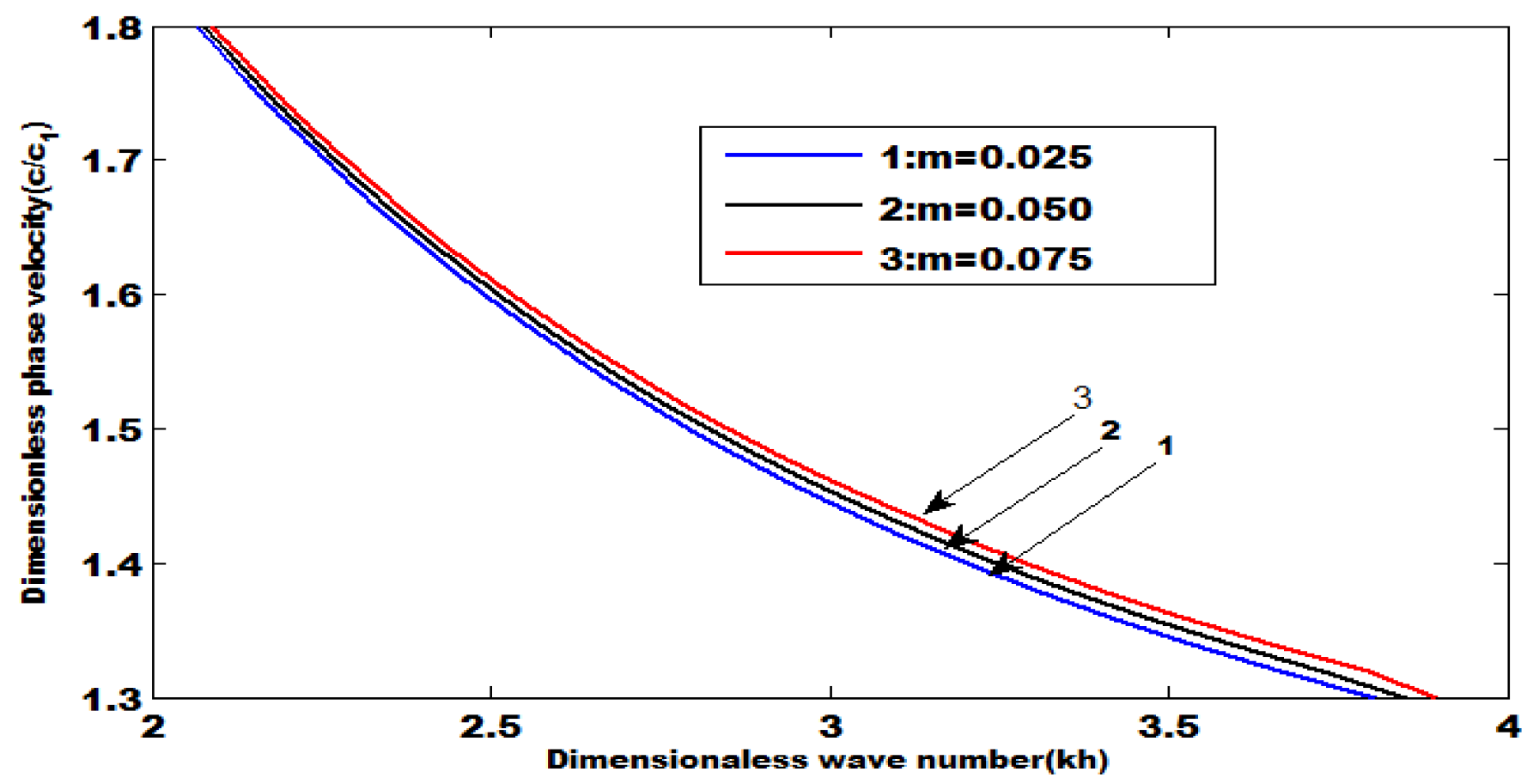

Figure 1. The phase velocity with wave number when $a_{1}=0.1, \alpha=0.2, \beta=0.1, h=0.04, p=0.01, a_{2}=0.2$.

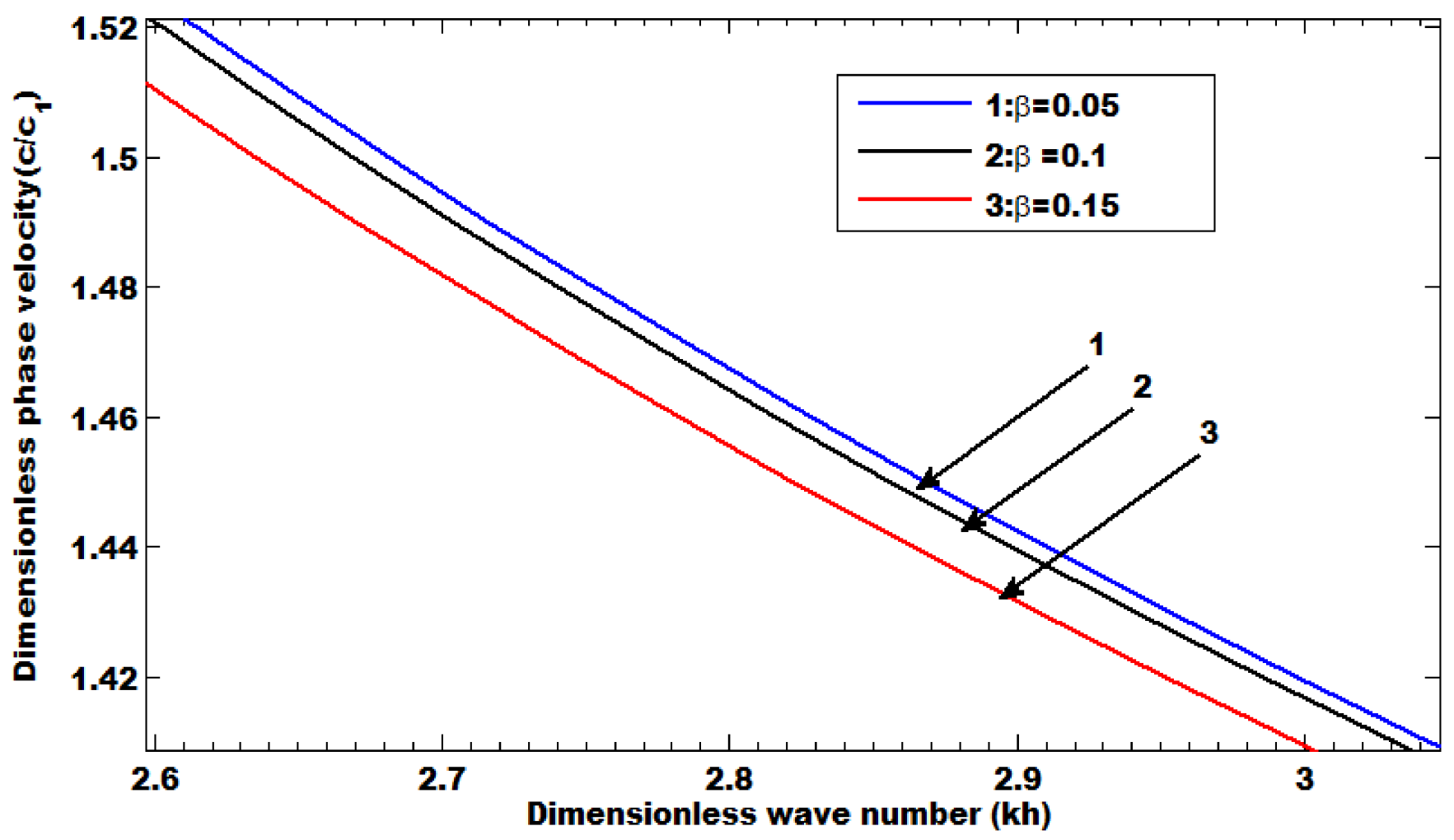

Figure 2. The phase velocity with wave number when $a_{1}=0.1, p=0.01, a_{2}=0.2, m=0.08, h=0.04, \alpha=0.2$. 


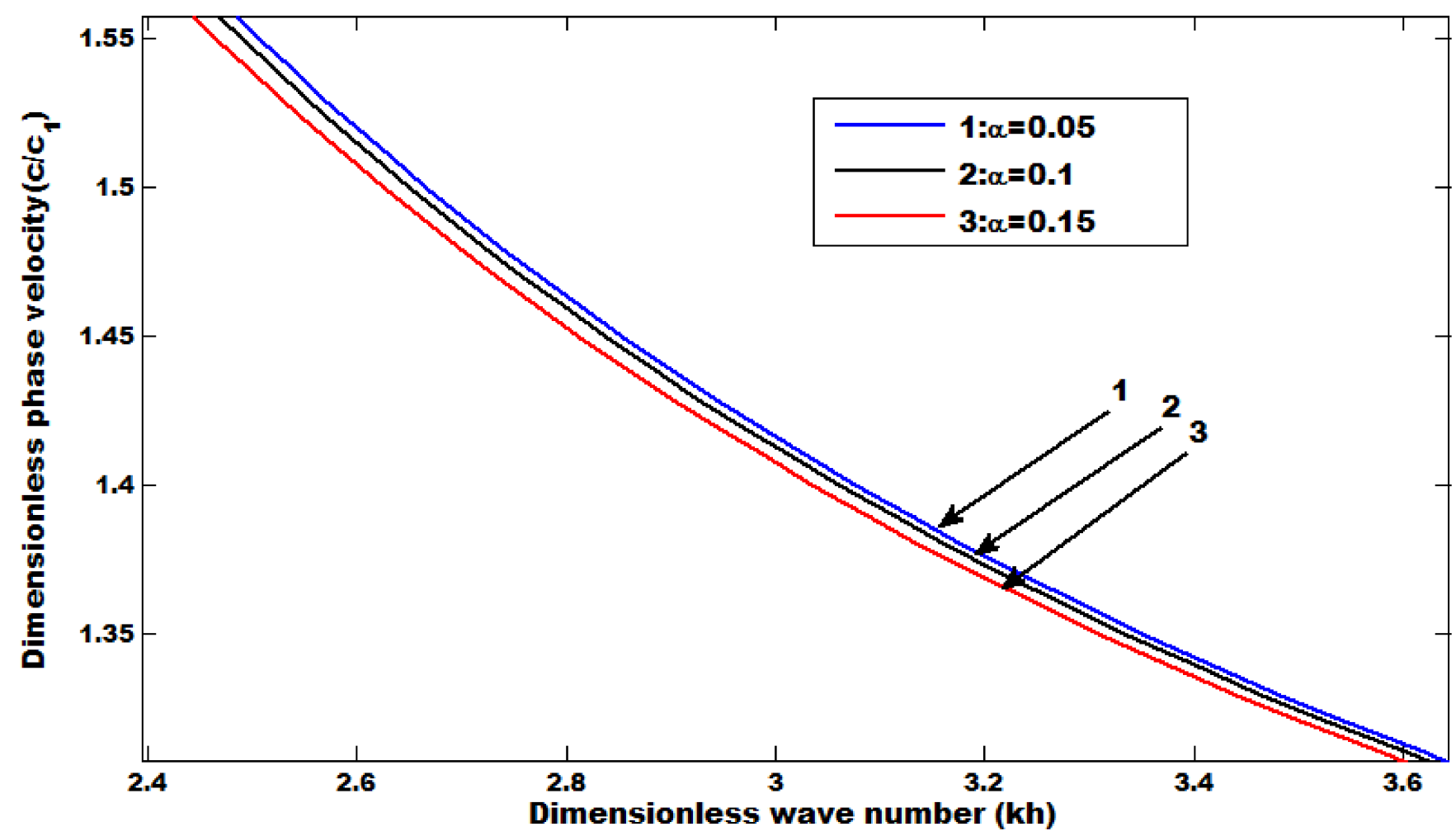

Figure 3. The phase velocity with wave number when $a_{1}=0.1, p=0.01, a_{2}=0.2, h=0.04, m=0.08, \beta=0.1$.

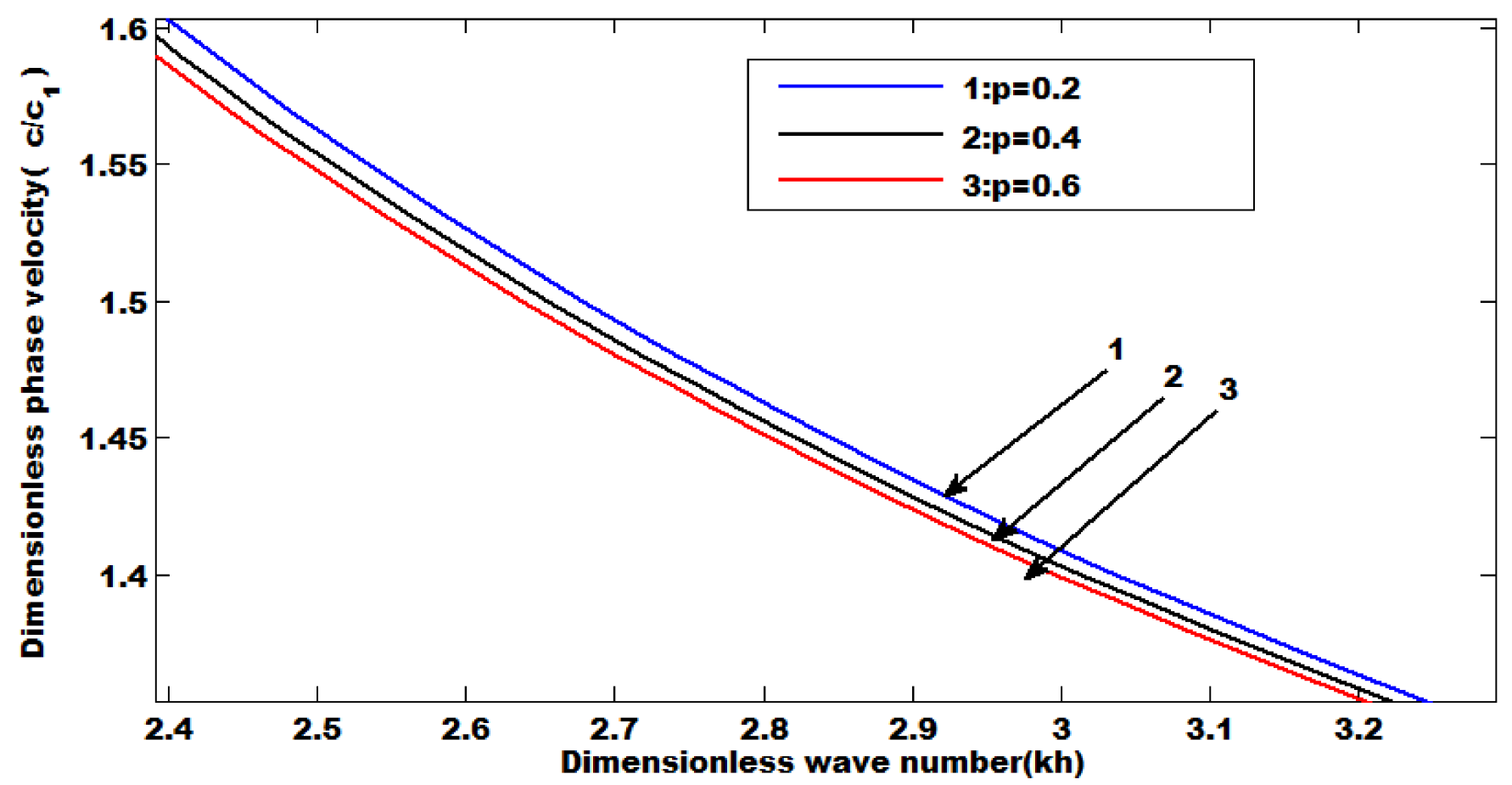

Figure 4. The phase velocity with wave number when $a_{1}=0.1, \beta=0.1, h=0.04, a_{2}=0.2, m=0.08, \alpha=0.2$. 


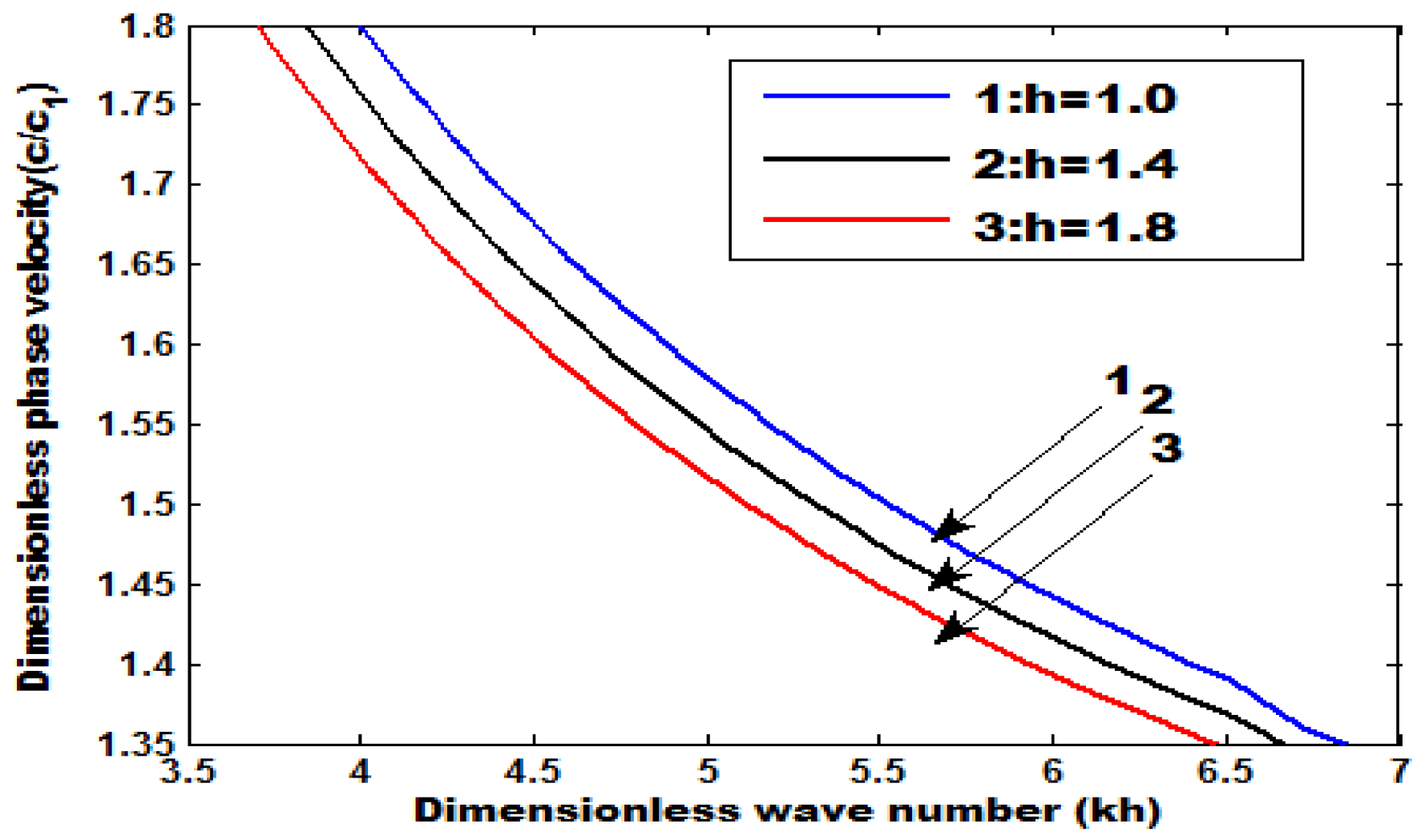

Figure 5. The phase velocity with wave number when $m=0.08, a_{1}=0.1, p=0.01, a_{2}=0.2, \alpha=0.2, \beta=0.1$.

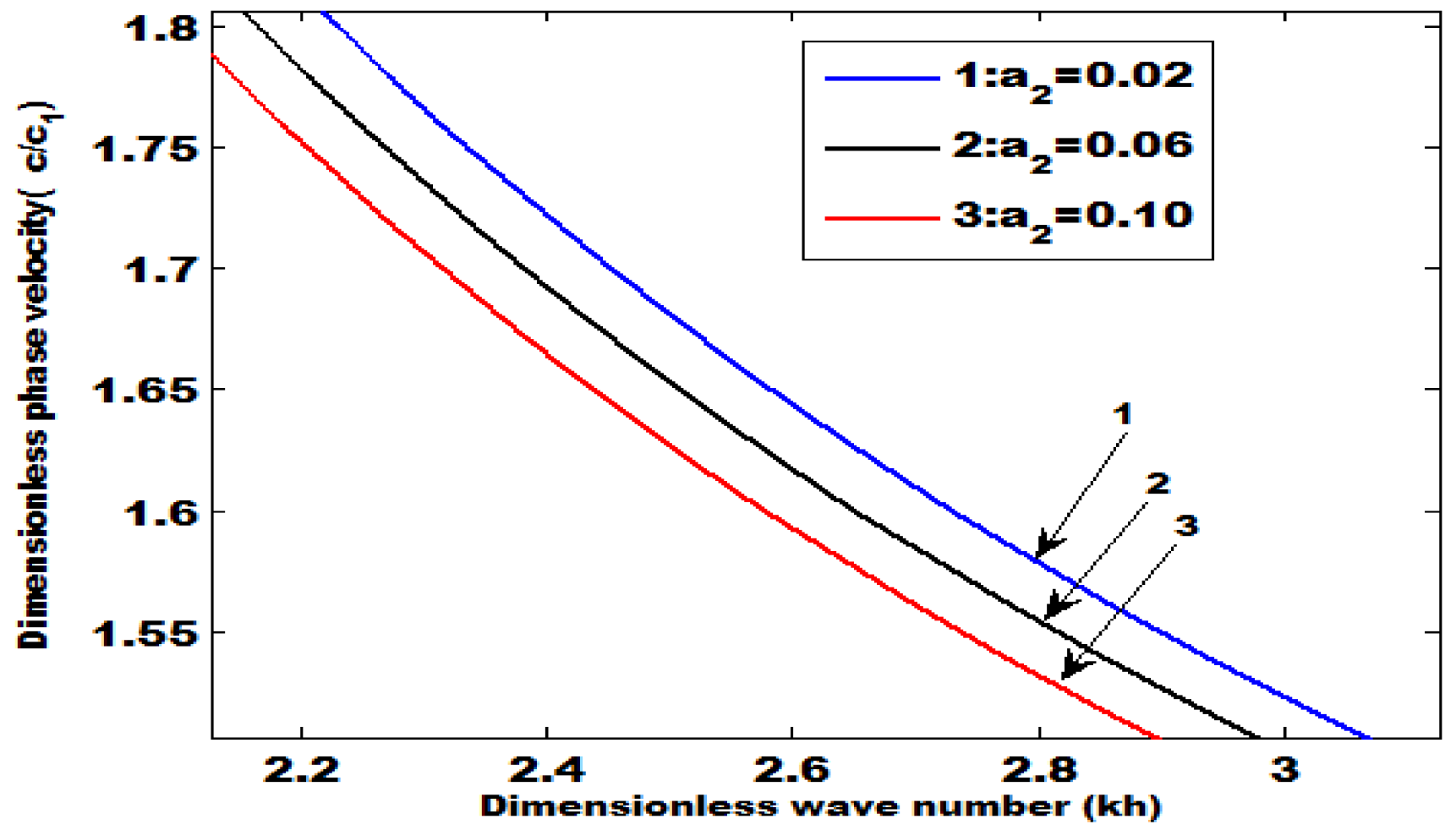

Figure 6. The phase velocity with wave number when $a_{1}=0.1, m=0.08, h=0.04, p=0.01, \alpha=0.2, \beta=0.1$. 


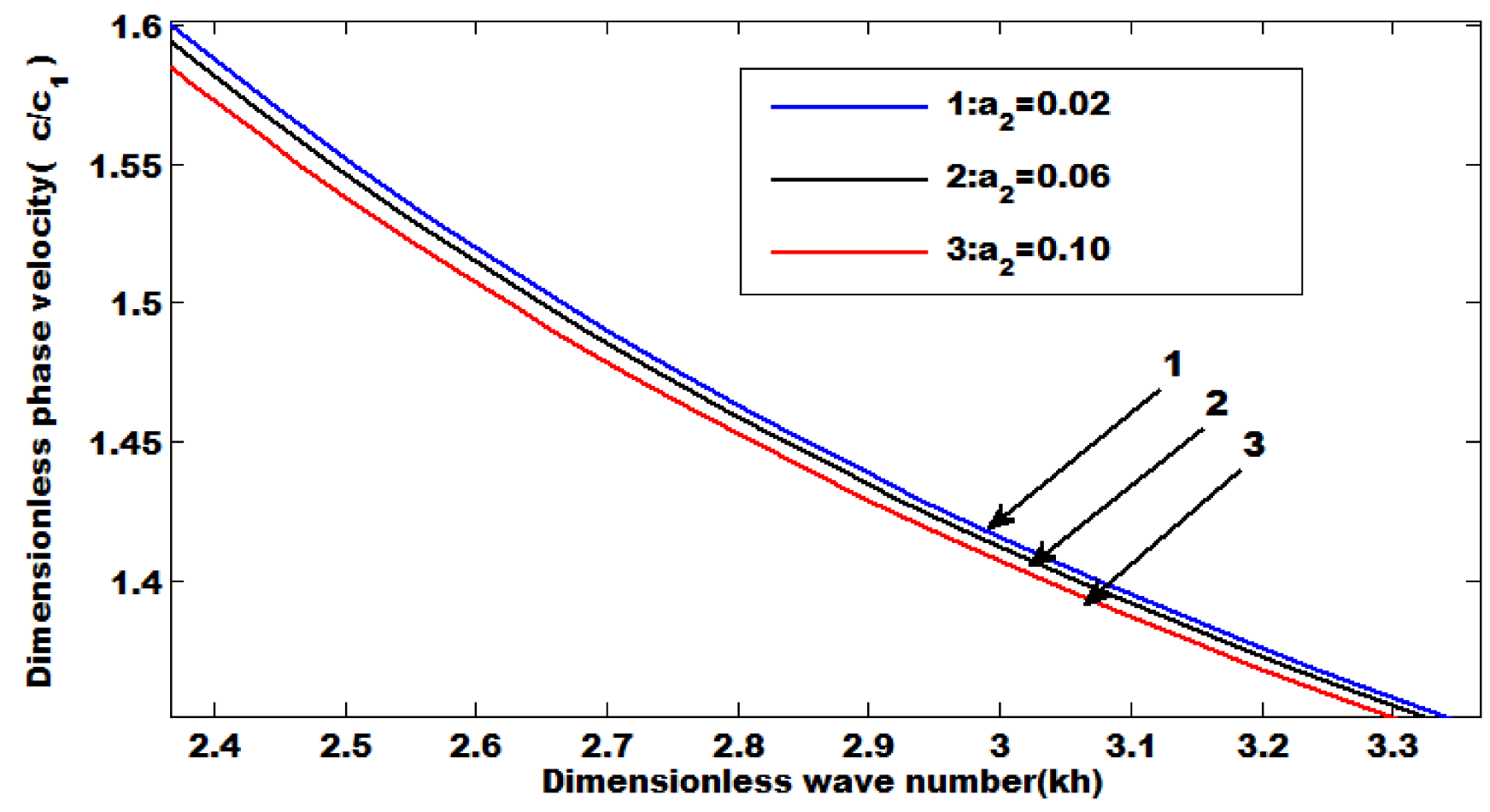

Figure 7. The phase velocity with wave number when $a_{1}=0, m=0.08, h=0.04, p=0.01, \alpha=0.2, \beta=0.1$.

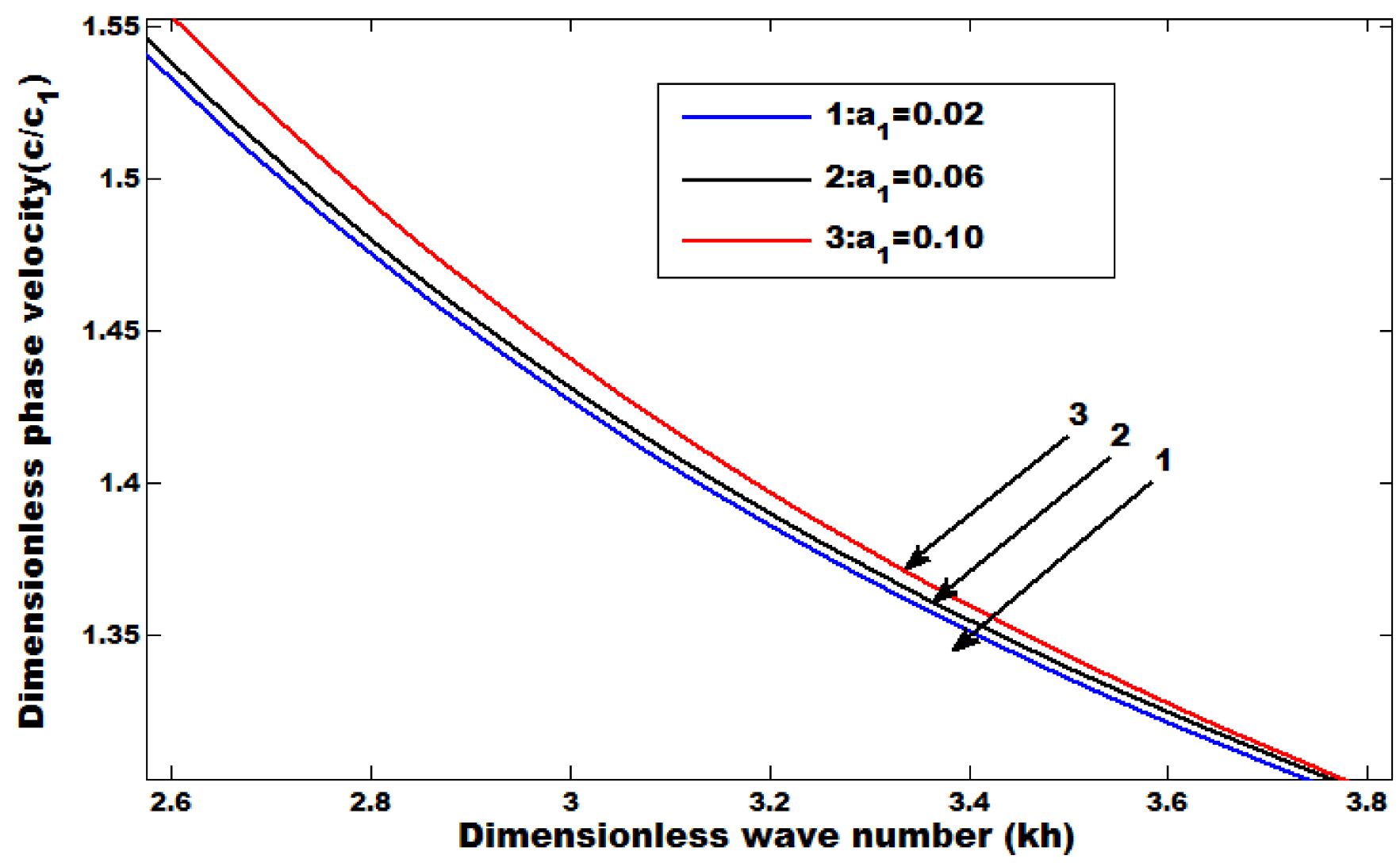

Figure 8. The phase velocity with wave number when $m=0.08, a_{2}=0.2, h=0.04, p=0.01, \alpha=0.2, \beta=0.1$. 


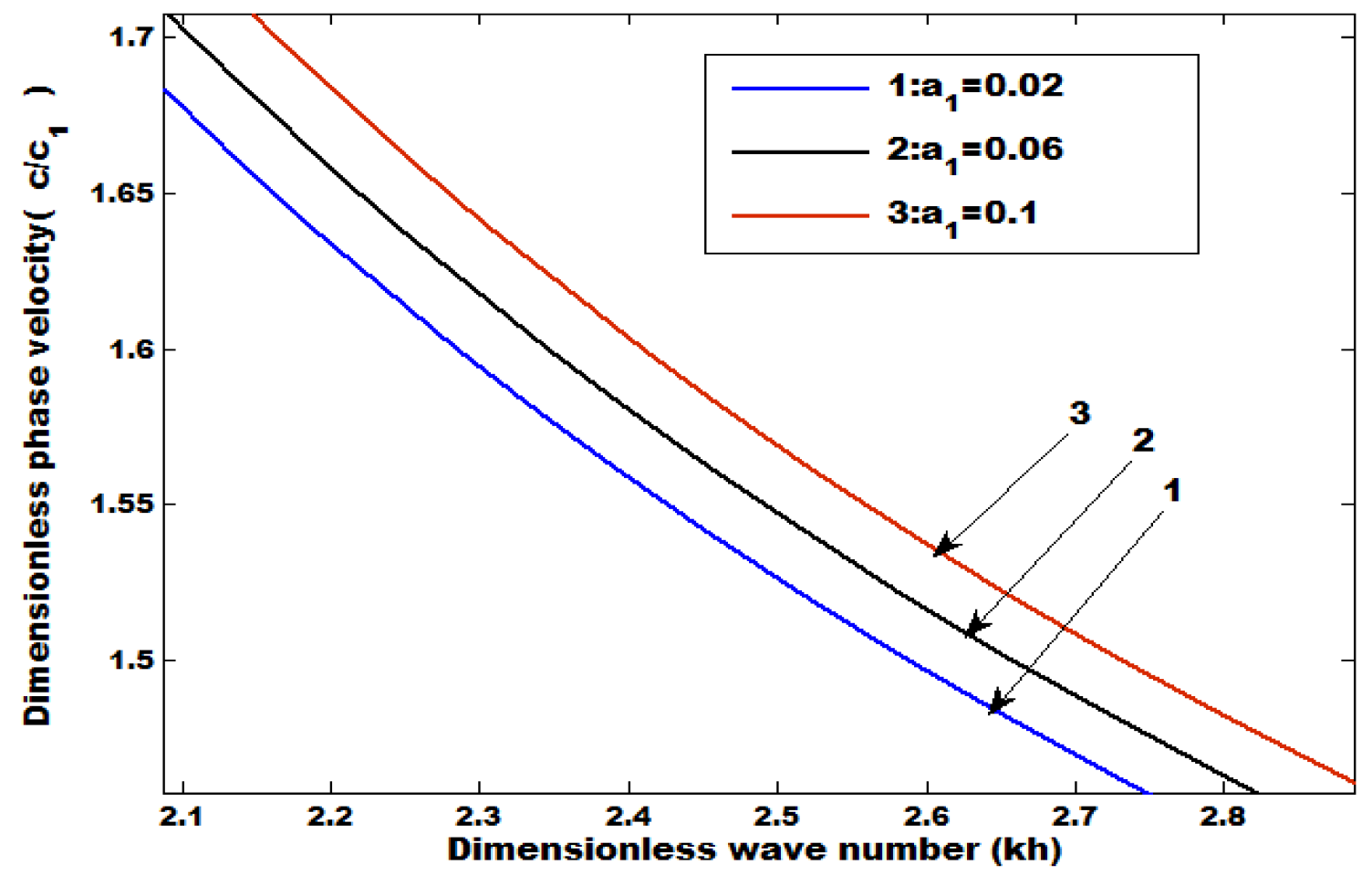

Figure 9. The phase velocity with wave number when $m=0.08, a_{2}=0, h=0.04, p=0.01, \alpha=0.2, \beta=0.1$.

Figures 10-12 are made to observe the effect of various parameters on group velocity. Figure 10 indicates the influence of coupling parameter on group velocity. As the coupling parameter increases, the group velocity also increases. Figures 11 and 12 describe the impact of heterogeneous parameters on group velocity. The group velocity declines as heterogeneous parameters increase.

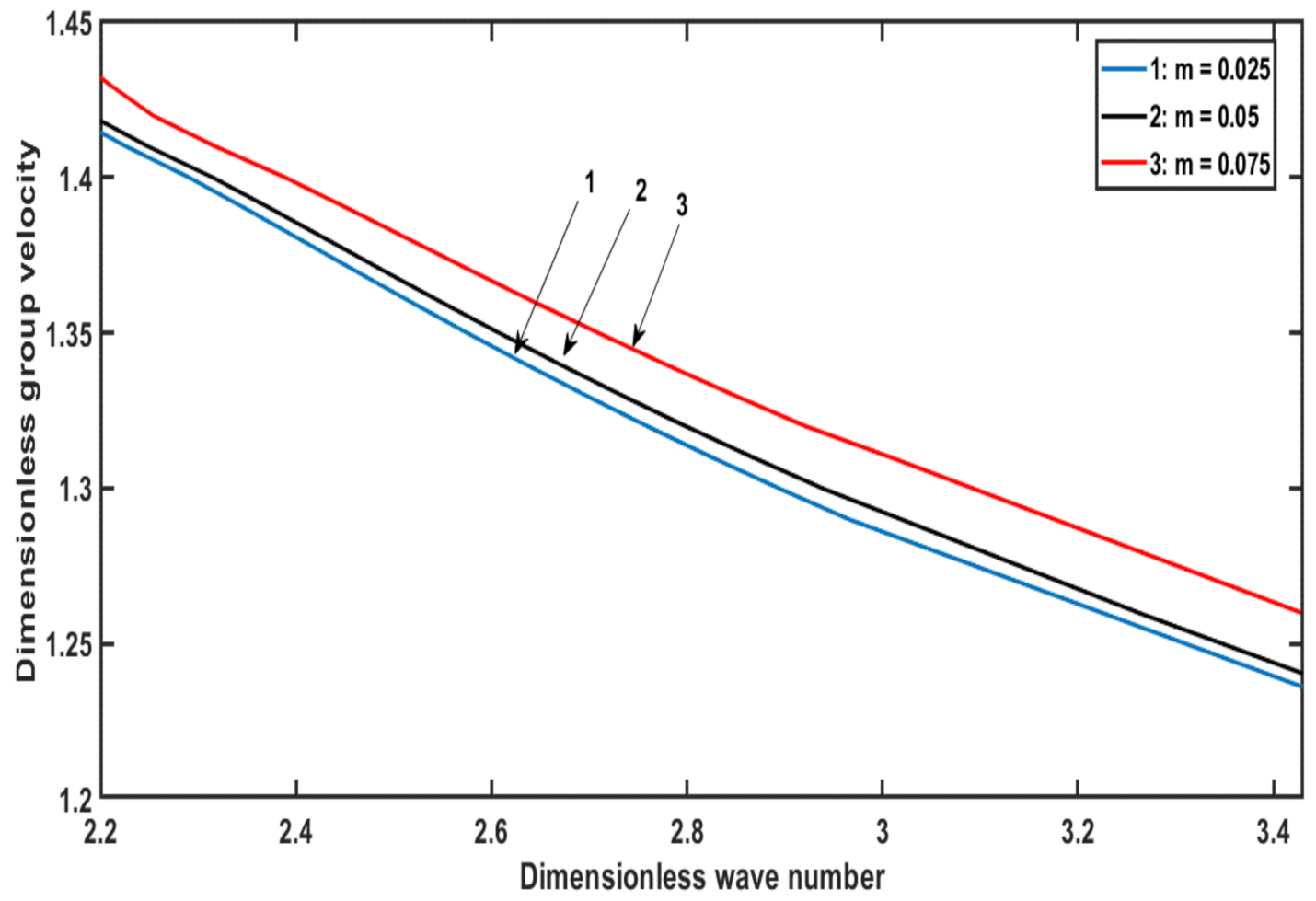

Figure 10. The group velocity with wave number when $a_{1}=0.1, p=0.01, a_{2}=0.2, h=0.01, \alpha=0.2, \beta=0.1$. 


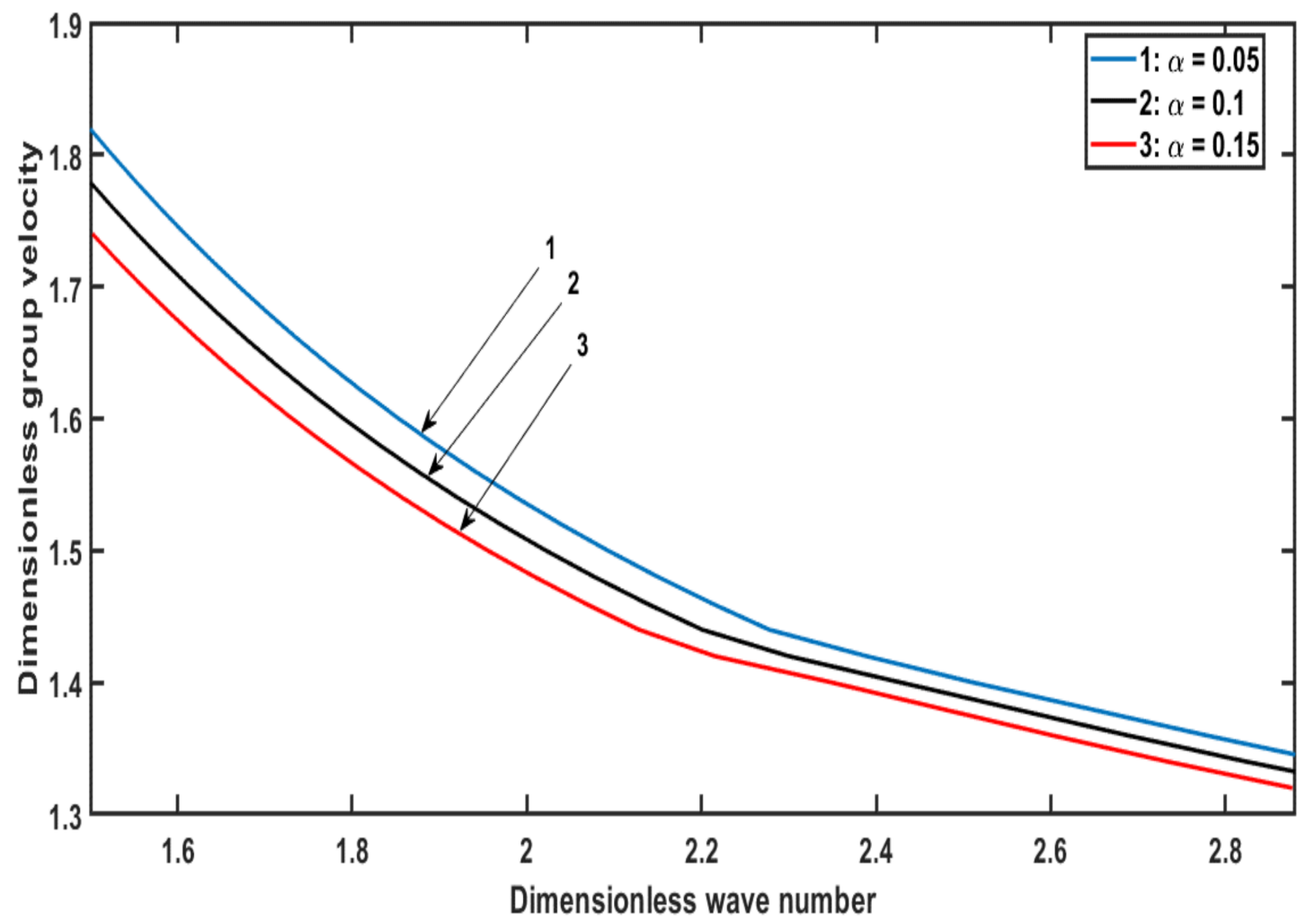

Figure 11. The group velocity with wave number when $a_{1}=0.1, p=0.01, m=0.08, h=0.04, a_{2}=0.2, \beta=0.1$.

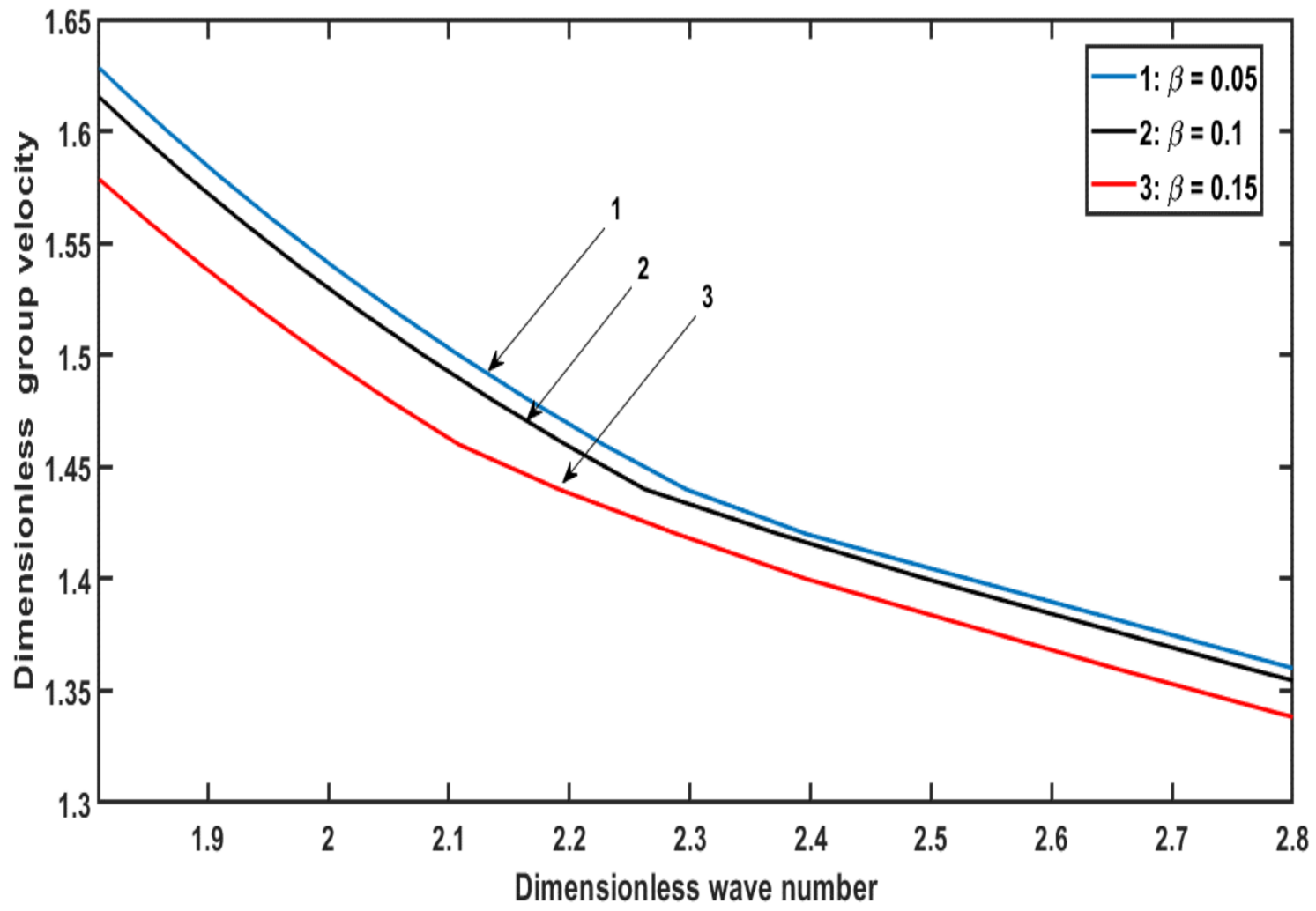

Figure 12. The group velocity with wave number when $a_{1}=0.1, p=0.01, m=0.08, a_{2}=0.2, \alpha=0.2, h=0.04$. 


\section{Conclusions}

The effect of initial stress on an $\mathrm{SH}$ wave in an anisotropic monoclinic layer over a heterogeneous half-space is discussed. The dispersion relation is gained numerically. The relationship between wave number and phase velocity was evaluated using graphs for dispersion equation. Whittaker's function is used to find the solution of the half-space. This study helps predict the behavior of an SH-wave under initial stress at mountain roots and continental region etc. The main findings are summarized as:

1. An increase in the coupling parameter and position parameter increases the phase velocity.

2. The phase velocity decreases by increasing the heterogeneous parameters.

3. The magnitude of phase velocity is greater in the absence of a common surface compared with the absence of an upper free surface.

Author Contributions: Conceptualization, A.A.K. and A.D.; Data curation, A.A.K. and M.R.-G.; Methodology, A.A.K., A.D. and M.M.A.; Supervision, A.A.K.; Writing-original draft, A.A.K. and A.D. All the authors contributed equally to this work. All authors have read and agreed to the published version of the manuscript.

Funding: Authors are grateful to the Deanship of Scientific Research, King Khalid University, Abha, Saudi Arabia, for financially supporting this work through the General Research Project under Grant no. R.G.P. $2 / 7 / 42$.

Institutional Review Board Statement: Not applicable.

Informed Consent Statement: Not applicable.

Data Availability Statement: Not applicable.

Acknowledgments: The authors would like to thank the reviewers for their careful reading and beneficial comments.

Conflicts of Interest: The authors declare no conflict of interest.

\section{References}

1. Jeffreys, H. The Effect on Love Waves of Heterogeneity in the Lower Layer. Geophys. J. Int. 1928, 2, 101-111. [CrossRef]

2. Bullen, K.E. The problem of the earth's density variation. Bull. Seismol. Soc. Am. 1940, 30, 235-250. [CrossRef]

3. Wilson, J.T. Surface waves in a heterogeneous medium. Bull. Seismol. Soc. Am. 1942, 32, 297-304. [CrossRef]

4. Dhua, S.; Chattopadhyay, A. Wave propagation in heterogeneous layers of the Earth. Waves Random Complex Media 2016, 26, 626-641. [CrossRef]

5. Alam, P.; Kundu, S.; Gupta, S. Dispersion study of SH-wave propagation in an irregular magneto-elastic anisotropic crustal layer over an irregular heterogeneous half-space. J. King Saud Univ.-Sci. 2018, 30, 301-310. [CrossRef]

6. Khan, A.A.; Umar, A.; Zaman, A. Rayleigh waves propagation in anisotropic layer superimposed a monoclinic medium. Indian J. Phys. 2019, 95, 449-457. [CrossRef]

7. Ilyashenko, A.V.; Kuznetsov, S.V. SH waves in anisotropic (monoclinic) media Z. Angew. Math. Phys. 2018, 17, 69. [CrossRef]

8. Singh, B.; Yadav, A.K. Plane wave in a rotating monoclinic magneto-thermoelastic medium. J. Eng. Phys. Phys. 2016, 89, 1393-1399.

9. Kumar, S.; Pal, P.C.; Majhi, S. Reflection and transmission of plane SH-waves through an anisotropic magnetoelastic layer sandwiched between two semi-infinite inhomogeneous viscoelastic half-spaces. Pure Appl. Geophy. 2015, 172, $2621-2634$. [CrossRef]

10. Zhang, Y.; Xu, G.; Zheng, Z. Terahertz waves propagation in an inhomogeneous plasma layer using the improved scattering-matrix method. Waves Random Complex Media 2020, 1-15. [CrossRef]

11. Rao, Q.; Xu, G.; Wang, P.; Zheng, Z. Study on the Propagation Characteristics of Terahertz Waves in Dusty Plasma with a Ceramic Substrate by the Scattering Matrix Method. Sensors 2021, 21, 263. [CrossRef] [PubMed]

12. Rao, Q.; Xu, G.; Wang, P.; Zheng, Z. Study of the Propagation Characteristics of Terahertz Waves in a Collisional and Inhomogeneous Dusty Plasma with a Ceramic Substrate and Oblique Angle of Incidence. Int. J. Antennas Propag. 2021, $2021,6625530$. [CrossRef]

13. Biot, M.A. The Influence of Initial Stress on Elastic Waves. J. Appl. Phys. 1940, 11, 522-530. [CrossRef]

14. Chatterjee, M.; Dhua, S.; Chattopadhyay, A.; Sahu, S.A. Reflection and Refraction for Three-Dimensional Plane Waves at the Interface between Distinct Anisotropic Half-Spaces under Initial Stresses. Int. J. Géoméch. 2016, 16, 04015099. [CrossRef] 
15. Singh, A.K.; Das, A.; Parween, Z.; Chattopadahyay, A. Influence of initial stress, irregularity and heterogeneity on Love-type wave propagation in double pre-stressed irregular layers lying over a pre-stressed half-space. J. Earth Syst. Sci. 2015, 124, 1457-1474. [CrossRef]

16. Abd-Alla, A.M.; Abo-Dahab, S.M.; Kilany, A.A. SV-waves incidence at interface between solid-liquid media under electromagnetic field and initial stress in the context of three thermoelastic theories. J. Therm. Stresses 2016, 39, 960-976. [CrossRef]

17. Verma, A.K.; Chattopadhyay, A.; Singh, A.K. Influence of Heterogeneity and Initial Stress on the Propagation of Rayleigh-type Wave in a Transversely Isotropic Layer. Procedia Eng. 2017, 173, 988-995. [CrossRef]

18. Khan, A.A.; Afzal, A. Influence of initial stress and gravity on refraction and reflection of SV wave at interface between two viscoelastic liquid under three thermoelastic theories. J. Braz. Soc. Mech. Sci. Eng. 2018, 40, 208. [CrossRef]

19. Tomar, S.K.; Kaur, J. Shear waves at a corrugated interface between anisotropic elastic and visco-elastic solid half-spaces. J. Seism. 2007, 11, 235-258. [CrossRef]

20. Whittaker, E.; Watson, G.N. A Course of Modern Analysis; Universal Book Stall: New Delhi, India, 1990.

21. Gubbin, D. Seismology and Plate Tectonics; Cambridge University Press: Cambridge, UK, 1990. 